\title{
Non-Candida Fungal Prosthetic Joint Infections
}

\author{
Christos Koutserimpas ${ }^{1}$, Ifigeneia Chamakioti ${ }^{2}$, Stylianos Zervakis ${ }^{3}{ }^{\mathbb{D}}$, Konstantinos Raptis ${ }^{1}$, \\ Kalliopi Alpantaki ${ }^{4}$, Diamantis P. Kofteridis ${ }^{5}$, Georgia Vrioni ${ }^{6}$ and George Samonis ${ }^{5, *}$
}

1 Department of Orthopaedics and Traumatology, “251” Hellenic Air Force General Hospital of Athens, 11525 Athens, Greece; chrisku91@hotmail.com (C.K.); kraptis1981@hotmail.gr (K.R.)

2 Emergency Department, “251” Hellenic Air Force General Hospital of Athens, 11525 Athens, Greece; ifigenia92@hotmail.com

3 Department of Cardiology, University Hospital of Heraklion, 71409 Heraklion, Greece; zervakis_st@hotmail.com

4 Department of Orthopaedics and Traumatology, "Venizeleion" General Hospital of Heraklion, 71409 Heraklion, Greece; apopaki@yahoo.gr

5 Department of Internal Medicine, University Hospital of Heraklion, 71500 Heraklion, Greece; kofteridisd@hotmail.com

6 Department of Microbiology, Medical School, National and Kapodistrian University of Athens, 11527 Athens, Greece; gvrioni@med.uoa.gr

* Correspondence: samonis@med.uoc.gr

check for updates

Citation: Koutserimpas, C.; Chamakioti, I.; Zervakis, S.; Raptis, K.; Alpantaki, K.; Kofteridis, D.P.; Vrioni, G.; Samonis, G. Non-Candida Fungal Prosthetic Joint Infections. Diagnostics 2021, 11, 1410. https:// doi.org/10.3390/diagnostics11081410

Academic Editors: Alfred O. Ankrah, Andor W. J. M. Glaudemans and Mike M. Sathekge

Received: 29 May 2021

Accepted: 3 August 2021

Published: 4 August 2021

Publisher's Note: MDPI stays neutral with regard to jurisdictional claims in published maps and institutional affiliations.

Copyright: (c) 2021 by the authors. Licensee MDPI, Basel, Switzerland. This article is an open access article distributed under the terms and conditions of the Creative Commons Attribution (CC BY) license (https:// creativecommons.org/licenses/by/ $4.0 /)$.
Abstract: Background: Fungal prosthetic joint infections (PJIs) are rare, especially those caused by non-Candida species. Treatment has not been fully elucidated, since a plethora of antifungal and surgical interventions have been proposed. This study represents an effort to clarify the optimal management of non-Candida fungal PJIs, by reviewing all relevant published cases. Methods: A thorough review of all existing non-Candida fungal PJIs in the literature was conducted. Data regarding demographics, responsible organisms, antifungal treatment (AFT), surgical intervention, time between initial arthroplasty and onset of symptoms, and time between onset of symptoms and firm diagnosis, as well as the infection's outcome, were evaluated. Results: Forty-two PJIs, in patients with mean age of 66.2 years, were found and reviewed. Aspergillus spp. were isolated in most cases $(10 ; 23.8 \%)$, followed by Coccidioides spp. (7; 16.7\%) and Pichia anomala $(5 ; 11.9 \%)$. Fluconazole was the preferred antifungal regimen (20 cases; $47.6 \%)$, followed by amphotericin B (18 cases; $42.9 \%$ ), while the mean AFT duration was 9.4 months (SD $=7.06)$. Two-stage revision arthroplasty (TSRA) was performed in 22 cases (52.4\%), with the mean time between stages being 5.2 months $(\mathrm{SD}=2.9)$. The mean time between initial joint implantation and onset of symptoms was 42.1 months ( $\mathrm{SD}=50.7$ ), while the mean time between onset of symptoms and diagnosis was 5.8 months $(\mathrm{SD}=14.3)$. Conclusions: Non-Candida fungal PJIs pose a clinical challenge, demanding a multidisciplinary approach. The present review has shown that combination of TSRA separated by a 3-6-month interval and prolonged AFT has been the standard of care in the studied cases.

Keywords: fungal infection; prosthetic joint infection; Aspergillus spp.; Coccidioides spp.; Pichia spp.; arthroplasty

\section{Introduction}

Joint arthroplasty represents a life-enhancing procedure, providing pain relief and restoration of function, and thereby improving patients' quality of life. Hip and knee arthroplasty rates are projected to reach 572,000 and 3.48 million, respectively, in the USA by 2030 [1]. Joint reconstruction surgery has evolved over time, encompassing minimally invasive surgical approaches, perioperative pain management and blood transfusion reduction protocols, and navigation or robotic systems, as well as new prosthetic materials [2-4]. Nevertheless, complications have not yet been eliminated. Prosthetic joint infections (PJIs) have serious implications on the patient's quality of life, and in some cases may prove fatal [5]. 
Fungal organisms are responsible for $\sim 1-2 \%$ of PJIs — even less in cases where non-Candida species are the cause [6]. Due to the rarity of these infections, no clear guidelines exist regarding management [7]. Currently, on the basis of limited data, a two-stage revision arthroplasty (TSRA) combined with prolonged antifungal treatment (AFT) is suggested [7,8].

The present study represents an effort, by reviewing all published cases of non-Candida fungal PJIs, to clarify the medical and surgical treatment options and their success-namely, the eradication of the infection, as well as the maintenance of the viability and functionality of the prosthetic joint, offering the patient the best possible quality of life. The present review takes into account the fact that it covers vast and epidemiologically diverse geographical areas, and that over the course of the long time period studied, medical therapeutic management has changed dramatically.

\section{Materials and Methods}

A meticulous electronic search of the PubMed and MEDLINE databases was conducted to identify all existing articles related to the treatment of non-Candida PJIs through to March 2021. Alone and/or in combination, the terms "fungal joint infection", "fungal prosthetic joint infection", "fungal knee arthroplasty infection", "fungal knee infection", "fungal shoulder arthroplasty infection", "fungal shoulder infection", "fungal elbow arthroplasty infection", "fungal elbow infection", "fungal hip arthroplasty infection", and "fungal hip infection" were searched. In addition, terms including each fungal species (e.g., "Aspergillus joint infection", "Coccidioides joint infection", etc.) were also searched.

The review was limited to papers published in English and in peer-reviewed journals. The data extracted from these studies included age, gender, affected joint, responsible non-Candida fungal organisms, duration and type of AFT, type of surgical intervention, use of antifungal-agent-loaded cement, time between initial arthroplasty and onset of symptoms, and definitive diagnosis (microscopy/culture/histopathology). Furthermore, the results of medical and surgical treatment, along with the follow-up of each case, were studied and evaluated.

In order to reach a conclusion of the success rates of each surgical treatment, all interventions that failed in each report were thoroughly evaluated. Cases not including surgical interventions were excluded from the assessment of the surgical success rate.

Treatment was considered successful if all signs and symptoms of the infection disappeared and no recurrence was observed during the follow-up period.

Finally, the patients' Charlson Comorbidity Index was calculated based on the information provided by each study.

Data were recorded and analyzed using Microsoft Excel 2019 (Microsoft Corporation, Redmond, WA, USA).

\section{Results}

A total of 42 cases (18; 43\% males), covering a 37-year period (1981-2018), were identified [9-34]. The studied population's mean age was 66.2 years (standard deviation $(\mathrm{SD})=13.9)$. The infected joint was the knee in 29 cases $(69 \%)$, the hip in $12(29 \%)$, and the elbow in $1(2 \%)$. In one case, the knee prosthetic joint infection was bilateral.

Patients' mean Charlson Comorbidity Index was 3.2 (SD = 1.6).

Furthermore, 26 cases (62\%) referred to primary joint reconstruction, while the remaining $16(38 \%)$ were revisions, with the mean number of revisions being $1.5(\mathrm{SD}=0.7)$. A total of 10 patients $(23.8 \%)$ were immunocompromised according to the available information from each report. The mean C-reactive protein (CRP) and erythrocyte sedimentation rate (ESR) at initial presentation were $58.8 \mathrm{mg} / \mathrm{L}(\mathrm{SD}=68.9)$ and $57 \mathrm{~mm} / \mathrm{h}(\mathrm{SD}=32.4)$, respectively. The mean time interval between the initial joint implantation surgery and the symptomatology of the onset of the infection was 42.1 months $(\mathrm{SD}=50.7)$, while the mean time interval between onset of symptoms and firm diagnosis was 5.8 months $(\mathrm{SD}=14.3)$. The mean follow-up was 44.2 months $(\mathrm{SD}=24.5)$. 
Regarding the causative fungal organisms, the most frequently isolated were Aspergillus spp. in 10 cases (23.8\%), followed by Coccidioides spp. in 7 (16.7\%) and Pichia anomala in $5(11.9 \%)$, while Acremonium spp., Alternaria spp., Cryptococcus neoformans, Histoplasma capsulatum, Pseudallescheria spp., and Rhodotorula spp. were each represented by 2 cases (4.8\%), and Aureobasidium spp., Malassezia spp., Phialemonium curvatum, Pithomyces spp., Sporothrix schenchii, Syncephalastrum racemosum, and Trichosporon asahii were represented by 1 case each (2.4\%). Furthermore, one case of unidentified mold was also reported (Table 1). In 12 cases (28.6\%), co-infection with bacteria was identified.

Regarding surgical intervention, a two-stage revision arthroplasty (TSRA) was performed in the majority of the reported patients (22 cases (52.4\%)), followed by "no surgical intervention" (8 cases (19\%)), resection arthroplasty (RA) and debridement (3 cases each $(7.1 \%))$, and one-stage revision arthroplasty (OSRA) and arthrodesis (2 cases each (4.8\%)) (Table 2). Furthermore, one case (2.4\%) of amputation was reported, while in one case $(2.4 \%)$ the type of surgical intervention was not reported (case 39 from Table 2).

The total success rate of any surgical intervention was $61 \%$. TSRA showed a $66.7 \%$ success rate; ORSA, arthrodesis, and debridement had success rates of $66.7 \%$ each; and RA had a 50\% success rate. In the amputation case, the infection was eradicated. All eight cases $(100 \%)$ that did not receive surgical intervention were considered, by the authors, as successfully treated only by medical means. It is of note that all of these eight patients were put on lifelong antifungal suppressive treatment (Table 2).

More specifically regarding TSRA, the mean time interval between the initial removal of the implants and the final re-implantation was 5.2 months $(\mathrm{SD}=2.9)$. Regarding the cement spacer used, in nine cases an antifungal regimen was impregnated in the cement; amphotericin $B$ was used in five of these cases, voriconazole in three, and itraconazole in one.

Regarding antifungal treatment (AFT), in 20 cases (47.6\%), a single agent was used; in $15(35.7 \%)$, two - either simultaneously or consecutively-while in $1(2.4 \%)$, more than two agents were used. In six cases the information regarding the specific antifungal drug was not available (cases 3, 4, 14, 15, 24, and 35 in Table 2). The mean duration of AFT was 9.4 months ( $\mathrm{SD}=7.06$ ), while it is of note that in eight cases a lifelong suppressive AFT was started (cases 17, 18, 19, 21, 22, 24, 25, and 27 in Table 2).

Fluconazole was the preferred agent in 20 cases $((47.6 \%)$, in $8(40 \%)$ as monotherapy), followed by amphotericin B in 18 cases $((42.9 \%)$, in $3(16.7 \%)$ as monotherapy), voriconazole in $8((19 \%)$, in $2(25 \%)$ as monotherapy), and itraconazole in $5((11.9 \%)$, in $3(60 \%)$ as monotherapy). Caspofungin and posaconazole, as monotherapy, were preferred in one case $(2.4 \%)$ each. The final outcome was successful in 33 cases $(78.6 \%)$. Regarding the most prevalent fungus, treatment was successful in $90 \%$ of Aspergillus spp., and in $100 \%$ of Coccidioides spp. and Pichia anomala.

The details of AFT are exhibited in Table 2. In the most frequently isolated Aspergillus spp. cases, the preferred AFT was fluconazole (5 cases $(50 \%)$, in $3(66.7 \%)$ as monotherapy), followed by amphotericin B (4 cases (40\%), in $2(50 \%)$ as monotherapy), and posaconazole, voriconazole, and caspofungin, which were given all as monotherapy in one case $(10 \%)$ each. The majority of patients with Coccidioides spp. Infections received fluconazole (4 cases $(57 \%)$, in $3(75 \%)$ as monotherapy), followed by amphotericin B (2 cases $(28.6 \%)$, in $1(50 \%)$ as monotherapy) and itraconazole (1 case (14.3\%) as monotherapy). Most patients with Pichia anomala were treated with fluconazole (5 cases $(100 \%)$, in $1(20 \%)$ as monotherapy), followed by amphotericin B ( 4 cases $(80 \%)$, none as monotherapy). 


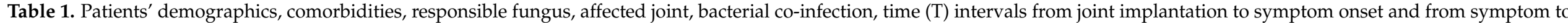
diagnosis, number of previous revisions in the same joint, C-reactive protein, (CRP), and erythrocyte sedimentation rate (ESR) at presentation.

\begin{tabular}{|c|c|c|c|c|c|c|c|c|c|c|c|c|c|c|}
\hline Case No & Year & Author & $\begin{array}{l}\text { Country } \\
\text { of Origin }\end{array}$ & Gender/Age & Fungus & Joint & $\begin{array}{c}\text { Co- } \\
\text { Infection }\end{array}$ & $\begin{array}{l}\text { CRP } \\
\mathrm{mg} / \mathrm{L}\end{array}$ & ESR mm/h & $\begin{array}{l}\text { Charlson } \\
\text { Comorbidity } \\
\text { Index }\end{array}$ & $\begin{array}{l}\text { Immunosuppressive } \\
\text { Medication and } \\
\text { Conditions }\end{array}$ & $\begin{array}{l}\text { Number } \\
\text { of } \\
\text { Previous } \\
\text { Revisions }\end{array}$ & $\begin{array}{l}\text { T from Im- } \\
\text { plantation } \\
\text { to Symp- } \\
\text { tomatology } \\
\text { (Months) }\end{array}$ & $\begin{array}{l}\text { T from } \\
\text { Symptom } \\
\text { Onset to } \\
\text { Diagnosis } \\
\text { (Months) }\end{array}$ \\
\hline 1 & 2014 & $\begin{array}{l}\text { Cheng-Yi } \\
\text { Wu et al. } \\
\text { [9] }\end{array}$ & Taiwan & $\mathrm{M} / 47$ & $\begin{array}{l}\text { Acremonium } \\
\text { spp. }\end{array}$ & Hip & $\begin{array}{l}\text { Penicillium } \\
\text { species }\end{array}$ & 16.17 & 127 & 3 & $\begin{array}{l}\text { Liver cirrhosis and } \\
\text { chronic HBV } \\
\text { infection }\end{array}$ & - & 120 & 2 \\
\hline 2 & 2018 & $\begin{array}{l}\text { Zhisen } \\
\text { Gao et al. } \\
\text { [10] }\end{array}$ & China & $\mathrm{F} / 52$ & $\begin{array}{l}\text { Acremonium } \\
\text { strictum }\end{array}$ & Knee & - & 0.348 & 17 & 1 & - & 1 & - & - \\
\hline 3 & 2018 & $\begin{array}{l}\text { Brown } \\
\text { et al. [11] }\end{array}$ & USA & $\mathrm{M} / 54$ & $\begin{array}{l}\text { Alternaria } \\
\text { spp. }\end{array}$ & Knee & - & 35 & 36 & 1 & - & - & - & - \\
\hline 4 & 2018 & $\begin{array}{l}\text { Brown } \\
\text { et al. [11] }\end{array}$ & USA & $\mathrm{F} / 55$ & $\begin{array}{l}\text { Alternaria } \\
\text { spp. }\end{array}$ & Knee & - & 35 & 36 & 1 & - & Yes (NA) & - & - \\
\hline 5 & 2016 & $\begin{array}{c}\text { Geng et al. } \\
\text { [12] }\end{array}$ & China & $\mathrm{F} / 63$ & $\begin{array}{l}\text { Aspergillus } \\
\text { spp. }\end{array}$ & Knee & - & 49.9 & 25 & 2 & - & - & - & - \\
\hline 6 & 2018 & $\begin{array}{l}\text { Zhisen } \\
\text { Gao et al. } \\
\text { [10] }\end{array}$ & China & $\mathrm{F} / 63$ & $\begin{array}{l}\text { Aspergillus } \\
\text { spp. }\end{array}$ & Knee & - & 4.99 & 25 & 2 & Diabetes Mellitus & 3 & - & - \\
\hline 8 & 1992 & $\begin{array}{l}\text { Austin } \\
\text { et al. [13] }\end{array}$ & USA & $\mathrm{M} / 80$ & $\begin{array}{l}\text { Aspergillus } \\
\text { fumigatus }\end{array}$ & Knee & - & - & 100 & 4 & $\begin{array}{c}\text { Megaloblastic } \\
\text { anemia, neutropenia, } \\
\text { immunosuppressive } \\
\text { therapy for the past } \\
\text { five years }\end{array}$ & - & 25 & 1 \\
\hline 9 & 2001 & $\begin{array}{l}\text { Baumann } \\
\text { et al. [14] }\end{array}$ & USA & $\mathrm{F} / 27$ & $\begin{array}{l}\text { Aspergillus } \\
\text { fumigatus }\end{array}$ & Knee & - & 37 & 55 & 2 & - & 1 & 53 & 0.1 \\
\hline 10 & 2011 & $\begin{array}{l}\text { Yilmaz } \\
\text { et al. [15] }\end{array}$ & Turkey & $\mathrm{M} / 81$ & $\begin{array}{l}\text { Aspergillus } \\
\text { fumigatus }\end{array}$ & Knee & - & 40 & 108 & 4 & - & - & 12 & 0.3 \\
\hline 11 & 2012 & $\begin{array}{l}\text { Hwang } \\
\text { et al. [16] }\end{array}$ & Korea & $\mathrm{F} / 74$ & $\begin{array}{l}\text { Aspergillus. } \\
\text { fumigatus }\end{array}$ & Knee & - & 63 & 32 & 4 & - & - & & \\
\hline
\end{tabular}


Table 1. Cont.

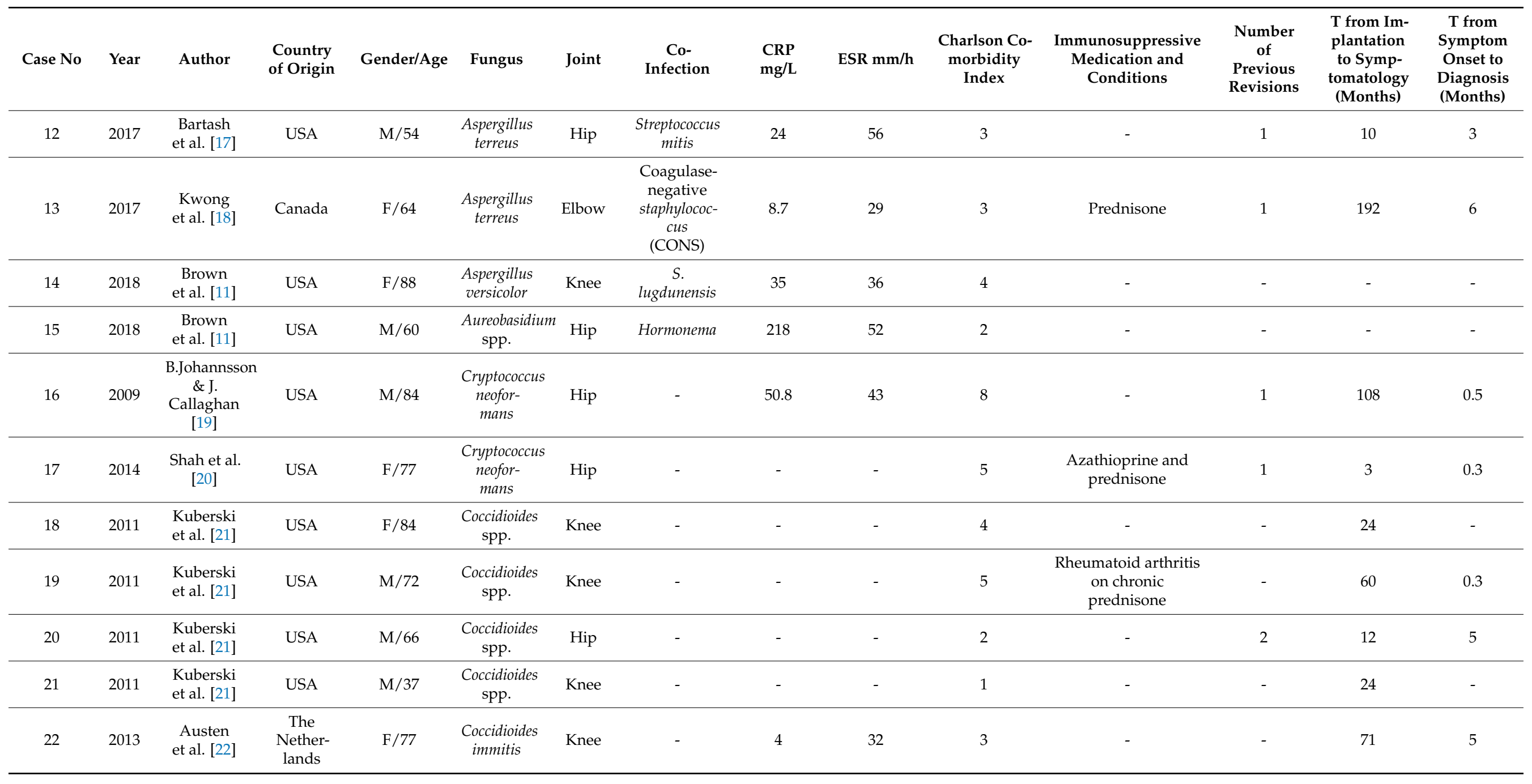


Table 1. Cont.

\begin{tabular}{|c|c|c|c|c|c|c|c|c|c|c|c|c|c|c|}
\hline Case No & Year & Author & $\begin{array}{l}\text { Country } \\
\text { of Origin }\end{array}$ & Gender/Age & Fungus & Joint & $\begin{array}{c}\text { Co- } \\
\text { Infection }\end{array}$ & $\begin{array}{l}\mathrm{CRP} \\
\mathrm{mg} / \mathrm{L}\end{array}$ & ESR mm/h & $\begin{array}{l}\text { Charlson Co- } \\
\text { morbidity } \\
\text { Index }\end{array}$ & $\begin{array}{l}\text { Immunosuppressive } \\
\text { Medication and } \\
\text { Conditions }\end{array}$ & $\begin{array}{c}\text { Number } \\
\text { of } \\
\text { Previous } \\
\text { Revisions }\end{array}$ & $\begin{array}{l}\mathrm{T} \text { from Im- } \\
\text { plantation } \\
\text { to Symp- } \\
\text { tomatology } \\
\text { (Months) }\end{array}$ & $\begin{array}{c}\text { T from } \\
\text { Symptom } \\
\text { Onset to } \\
\text { Diagnosis } \\
\text { (Months) }\end{array}$ \\
\hline 23 & 2015 & $\begin{array}{l}\text { Arbeloa- } \\
\text { Gutierrez } \\
\text { et al. [23] }\end{array}$ & Spain & $\mathrm{M} / 74$ & $\begin{array}{l}\text { Coccidioides } \\
\text { spp. }\end{array}$ & Knee & - & - & 132 & 3 & $\begin{array}{l}\text { Adrenal insufficiency, } \\
\text { corticosteroids }\end{array}$ & - & 84 & - \\
\hline 24 & 2018 & $\begin{array}{l}\text { Brown } \\
\text { et al. [11] }\end{array}$ & USA & $\mathrm{M} / 89$ & $\begin{array}{l}\text { Coccidioides } \\
\text { immitis }\end{array}$ & Hip & - & 218 & 52 & 4 & - & - & - & - \\
\hline 25 & 1998 & $\begin{array}{l}\text { Fowler } \\
\text { et al. [24] }\end{array}$ & USA & $\mathrm{F} / 84$ & $\begin{array}{l}\text { Histoplasma } \\
\text { capsula- } \\
\text { tum }\end{array}$ & Hip & - & - & 51 & 5 & $\begin{array}{c}\text { Prednisone- } \\
\text { dependent } \\
\text { polymyalgia } \\
\text { rheumatica, } \\
\text { granulomatous } \\
\text { disease }\end{array}$ & 2 & 96 & 60 \\
\hline 26 & 2017 & $\begin{array}{l}\text { Nowbakht } \\
\text { et al. [25] }\end{array}$ & USA & $\mathrm{M} / 77$ & $\begin{array}{l}\text { Histoplasma } \\
\text { capsula- } \\
\text { tum }\end{array}$ & Knee & $\begin{array}{l}\text { Group B } \\
\text { Streptococci }\end{array}$ & - & - & 5 & - & 3 & 8 & 0.3 \\
\hline 27 & 2016 & $\begin{array}{l}\text { Leylabadlo } \\
\text { et al. [26] }\end{array}$ & Iran & $\mathrm{F} / 59$ & $\begin{array}{l}\text { Malassezia } \\
\text { spp. }\end{array}$ & Knee & - & 2.33 & - & 1 & - & - & 0.7 & - \\
\hline 28 & 2016 & $\begin{array}{l}\text { Geng et al. } \\
\text { [12] }\end{array}$ & China & $\mathrm{F} / 76$ & $\begin{array}{l}\text { Mould } \\
\text { (unidenti- } \\
\text { fied) }\end{array}$ & Knee & $\begin{array}{l}\text { Coagulase- } \\
\text { negative } \\
\text { staphylococ- } \\
\text { cus }\end{array}$ & 65 & 80 & 4 & - & - & 8 & - \\
\hline 29 & 2012 & $\begin{array}{l}\text { Anagnostakose } \\
\text { al. [27] }\end{array}$ & Germany & $\mathrm{M} / 64$ & $\begin{array}{l}\text { Phialemonium } \\
\text { curvatum }\end{array}$ & Knee & - & $>20$ & - & 2 & - & 1 & - & - \\
\hline 30 & 2012 & $\begin{array}{l}\text { Hwang } \\
\text { et al. [16] }\end{array}$ & Korea & $\mathrm{F} / 49$ & $\begin{array}{c}\text { Pichia } \\
\text { anomala }\end{array}$ & Knee & - & 48 & 42 & 1 & - & - & - & \\
\hline 31 & 2015 & $\begin{array}{l}\text { Q.-J. Wang } \\
\text { et al. [28] }\end{array}$ & China & $\mathrm{M} / 68$ & $\begin{array}{c}\text { Pichia } \\
\text { anomala }\end{array}$ & Knee & - & 48 & 38 & 2 & - & - & & \\
\hline 32 & 2018 & $\begin{array}{l}\text { Hwang } \\
\text { et al. [16] }\end{array}$ & Korea & $\mathrm{F} / 73$ & $\begin{array}{c}\text { Pichia } \\
\text { anomala }\end{array}$ & Knee & & 41 & 45 & 4 & - & - & & \\
\hline 33 & 2018 & $\begin{array}{l}\text { Hwang } \\
\text { et al. [16] }\end{array}$ & Korea & F/ 66 & $\begin{array}{c}\text { Pichia } \\
\text { anomala }\end{array}$ & $\begin{array}{l}\text { Knee } \\
\text { (bilat- } \\
\text { eral) }\end{array}$ & C. lusitaniae & 15 & 18 & 3 & - & - & & \\
\hline 34 & 2018 & $\begin{array}{l}\text { Hwang } \\
\text { et al. [16] }\end{array}$ & Korea & $\mathrm{F} / 70$ & $\begin{array}{l}\text { Pichia } \\
\text { anomala }\end{array}$ & Knee & - & 32 & 93 & 4 & - & - & & \\
\hline
\end{tabular}


Table 1. Cont.

\begin{tabular}{|c|c|c|c|c|c|c|c|c|c|c|c|c|c|c|}
\hline Case No & Year & Author & $\begin{array}{l}\text { Country } \\
\text { of Origin }\end{array}$ & Gender/Age & Fungus & Joint & $\begin{array}{c}\text { Co- } \\
\text { Infection }\end{array}$ & $\begin{array}{l}\mathrm{CRP} \\
\mathrm{mg} / \mathrm{L}\end{array}$ & ESR mm/h & $\begin{array}{l}\text { Charlson Co- } \\
\text { morbidity } \\
\text { Index }\end{array}$ & $\begin{array}{l}\text { Immunosuppressive } \\
\text { Medication and } \\
\text { Conditions }\end{array}$ & $\begin{array}{c}\text { Number } \\
\text { of } \\
\text { Previous } \\
\text { Revisions }\end{array}$ & $\begin{array}{l}\text { T from Im- } \\
\text { plantation } \\
\text { to Symp- } \\
\text { tomatology } \\
\text { (Months) }\end{array}$ & $\begin{array}{c}\text { T from } \\
\text { Symptom } \\
\text { Onset to } \\
\text { Diagnosis } \\
\text { (Months) }\end{array}$ \\
\hline 35 & 2018 & $\begin{array}{l}\text { Brown } \\
\text { et al. [11] }\end{array}$ & USA & $\mathrm{F} / 77$ & $\begin{array}{l}\text { Pithomyces } \\
\text { spp. }\end{array}$ & Hip & $\begin{array}{l}\text { Propionobacterium } \\
\text { acnes }\end{array}$ & 218 & 52 & 3 & - & - & - & - \\
\hline 36 & 2011 & $\begin{array}{l}\text { Gottesman- } \\
\text { Yekutieli } \\
\text { et al. [29] }\end{array}$ & Israel & $\mathrm{F} / 56$ & $\begin{array}{l}\text { Pseudallescheria } \\
\text { boydii }\end{array}$ & ${ }^{a}$ Hip & - & 169 & - & 2 & $\begin{array}{l}\text { Chronic joint disease } \\
\text { on prednisone and } \\
\text { methotrexate }\end{array}$ & - & 12 & 12 \\
\hline 37 & 2011 & $\begin{array}{l}\text { Lackner } \\
\text { et al. [30] }\end{array}$ & Austria & $\mathrm{M} / 61$ & $\begin{array}{l}\text { Pseudallescheria } \\
\text { apiosperma }\end{array}$ & ${ }^{a}$ Knee & - & 200 & 102 & 2 & - & - & 1.2 & 1 \\
\hline 38 & 2018 & $\begin{array}{l}\text { Hwang } \\
\text { et al. [16] }\end{array}$ & Korea & $\mathrm{F} / 75$ & $\begin{array}{l}\text { Rhodotorula } \\
\text { minuta }\end{array}$ & Knee & MRSA & 29 & 71 & 4 & - & - & & \\
\hline 39 & 2008 & $\begin{array}{l}\text { Savini et al. } \\
\text { [31] }\end{array}$ & Italy & $\mathrm{F} / 41$ & $\begin{array}{l}\text { Rhodotorula } \\
\text { mucilagi- } \\
\text { nosa }\end{array}$ & Hip & - & - & - & 6 & $\begin{array}{c}\text { Human } \\
\text { immunodeficiency } \\
\text { virus }\end{array}$ & 1 & & 0.5 \\
\hline 40 & 1995 & $\begin{array}{c}\text { DeHart } \\
\text { [32] }\end{array}$ & USA & $\mathrm{M} / 56$ & $\begin{array}{c}\text { Sporothrix } \\
\text { schenckii }\end{array}$ & Knee & - & - & - & 1 & & - & - & \\
\hline 42 & 2015 & $\begin{array}{c}\text { Zuo et al. } \\
\text { [34] }\end{array}$ & China & $\mathrm{F} / 73$ & $\begin{array}{l}\text { Trichosporon } \\
\text { asahii }\end{array}$ & Knee & - & 27.2 & 32 & 5 & & & 1 & 0.5 \\
\hline
\end{tabular}


Table 2. Surgical and antifungal treatment, follow-up, and infection outcome of the reported cases. ST: surgical treatment; TSRA: two-stage revision arthroplasty; OSRA: one-stage revision arthroplasty; AFT: antifungal treatment; LS: lifelong suppression; NS: no surgery; RA: resection arthroplasty; NA: not available.

\begin{tabular}{|c|c|c|c|c|c|c|c|}
\hline Case & ST & $\begin{array}{l}\text { Time between } \\
\text { Stages in TSRA } \\
\text { (Months) }\end{array}$ & $\begin{array}{l}\text { Antifungal } \\
\text { Regimen in } \\
\text { Cement }\end{array}$ & $\begin{array}{c}\text { Antifungal } \\
\text { Treatment (AFT) }\end{array}$ & $\begin{array}{l}\text { Total Duration } \\
\text { of AFT } \\
\text { (Months) }\end{array}$ & $\begin{array}{l}\text { Follow-Up } \\
\text { (Months) }\end{array}$ & Outcome \\
\hline 1 & TSRA & 4 & - & Fluconazole & 15 & 12 & Success \\
\hline 2 & TSRA & 9 & Voriconazole & $\begin{array}{l}\text { Voriconazole, } \\
\text { Fluconazole }\end{array}$ & 6.5 & 30 & Success \\
\hline 3 & TSRA & 6 & $\begin{array}{c}\text { Amphotericin } \\
\text { B }\end{array}$ & NA & - & 60 & - \\
\hline 4 & TSRA & 6 & $\begin{array}{c}\text { Amphotericin } \\
\text { B }\end{array}$ & NA & - & 60 & - \\
\hline 5 & TSRA & 7 & - & Fluconazole & 7 & 62 & Success \\
\hline 6 & TSRA & 7 & - & Fluconazole & 8.5 & 80 & Success \\
\hline 7 & $\begin{array}{l}\text { TSRA }(2 \times \text { spacer } \\
\text { exchange before } \\
\text { final implantation })\end{array}$ & 14 & - & Fluconazole & 3 & 51 & Failure \\
\hline 8 & RA & - & - & Amphotericin B & 3 & - & Success \\
\hline 9 & TSRA & 3.5 & - & $\begin{array}{l}\text { Amphotericin B, } \\
\text { Fluconazole }\end{array}$ & 10.5 & 60 & Success \\
\hline 10 & TSRA & 4 & - & Amphotericin B & 1.5 & 48 & Success \\
\hline 11 & TSRA & 3 & - & $\begin{array}{l}\text { Amphotericin B, } \\
\text { Fluconazole }\end{array}$ & - & 67 & Success \\
\hline 12 & TSRA & 4 & Voriconazole & Posaconzole & $>1$ & - & - \\
\hline 13 & $\begin{array}{c}\text { RA }(2 \times \text { TSRA } \\
\text { failed prior to RA })\end{array}$ & - & - & $\begin{array}{l}\text { Voriconazole, } \\
\text { Caspofungin }\end{array}$ & - & & Success \\
\hline 14 & TSRA & 6 & $\begin{array}{c}\text { Amphotericin } \\
\text { B }\end{array}$ & NA & - & 60 & - \\
\hline 15 & NS (no surgery) & - & - & $\begin{array}{l}\text { AFT Suppression } \\
\text { (NA) }\end{array}$ & - & 60 & Success \\
\hline 16 & RA & - & - & Amphotericin B & - & 10 & Failure \\
\hline 17 & NS & - & - & $\begin{array}{l}\text { Amphotericin B, } \\
\text { fluconazole }\end{array}$ & LS & 12 & Success \\
\hline 18 & Arthrodesis & - & - & Amphotericin B & LS & 48 & Success \\
\hline 19 & NS & - & - & $\begin{array}{l}\text { Amphotericin B, } \\
\text { Fluconazole }\end{array}$ & LS & 48 & Success \\
\hline 20 & $\begin{array}{l}\text { OSRA }(\times 2, \text { failed } \\
\text { the first time })\end{array}$ & - & - & Fluconazole & 17 & 12 & Success \\
\hline 21 & NS & - & - & Fluconazole & LS & 96 & Success \\
\hline 22 & NS & - & - & Fluconazole & LS & 6 & Success \\
\hline 23 & Arthrodesis & - & $\begin{array}{c}\text { Amphotericin } \\
\text { B }\end{array}$ & Itraconazole & - & 6 & Success \\
\hline 24 & NS & - & - & - & LS & 60 & Success \\
\hline 25 & Debridement & - & & Itraconazole & LS & 36 & Success \\
\hline 26 & TSRA & 9 & Voriconazole & Itraconazole & 24 & 24 & Success \\
\hline 27 & NS & - & - & $\begin{array}{l}\text { Amphotericin B, } \\
\text { Fluconazole, } \\
\text { Voriconazole }\end{array}$ & LS & - & Success \\
\hline 28 & TSRA & 3 & - & Fluconazole & 2.5 & 44 & Success \\
\hline 29 & OSRA & - & - & Voriconazole & 6 & 5 & Success \\
\hline 30 & TSRA & 2.5 & - & $\begin{array}{l}\text { Amphotericin B, } \\
\text { Fluconazole }\end{array}$ & - & 45 & Success \\
\hline
\end{tabular}


Table 2. Cont.

\begin{tabular}{|c|c|c|c|c|c|c|c|}
\hline Case & ST & $\begin{array}{l}\text { Time between } \\
\text { Stages in TSRA } \\
\text { (Months) }\end{array}$ & $\begin{array}{l}\text { Antifungal } \\
\text { Regimen in } \\
\text { Cement }\end{array}$ & $\begin{array}{c}\text { Antifungal } \\
\text { Treatment (AFT) }\end{array}$ & $\begin{array}{c}\text { Total Duration } \\
\text { of AFT } \\
\text { (Months) }\end{array}$ & $\begin{array}{l}\text { Follow-Up } \\
\text { (Months) }\end{array}$ & Outcome \\
\hline 31 & TSRA & 6 & $\begin{array}{c}\text { Amphotericin } \\
\text { B }\end{array}$ & Fluconazole & 3 & 65 & Success \\
\hline 32 & TSRA & 2.5 & - & $\begin{array}{l}\text { Amphotericin B, } \\
\text { Fluconazole }\end{array}$ & - & 67 & Success \\
\hline 33 & TSRA & 2 & - & $\begin{array}{l}\text { Amphotericin B, } \\
\text { Fluconazole }\end{array}$ & - & 35 & Success \\
\hline 34 & TSRA & 4 & - & $\begin{array}{l}\text { Amphotericin B, } \\
\text { Fluconazole }\end{array}$ & - & 67 & Success \\
\hline 35 & Debridement & - & - & NA & - & 60 & Success \\
\hline 36 & TSRA & 6.5 & Itraconazole & Voriconazole & 10 & 24 & Success \\
\hline 37 & $\begin{array}{l}\text { Amputation }(1 \times \\
\text { RA and } 1 \times \\
\text { arthrodesis both } \\
\text { failed) }\end{array}$ & - & - & $\begin{array}{l}\text { Itraconazole, } \\
\text { Voriconazole }\end{array}$ & 5.5 & 96 & Success \\
\hline 38 & TSRA & 2.5 & - & $\begin{array}{l}\text { Amphotericin B, } \\
\text { Fluconazole }\end{array}$ & - & 26 & Failure \\
\hline 39 & NA & - & - & Amphotericin B & $>0.75$ & - & NA \\
\hline 40 & NS & - & - & $\begin{array}{l}\text { Amphotericin B, } \\
\text { Itraconazole }\end{array}$ & 24 & 30 & Success \\
\hline 41 & TSRA & 2.5 & - & $\begin{array}{l}\text { Amphotericin B, } \\
\text { Voriconazole }\end{array}$ & - & 36 & Success \\
\hline 42 & Debridement & - & - & $\begin{array}{l}\text { Amphotericin B, } \\
\text { Voriconazole }\end{array}$ & 12 & 26 & Failure \\
\hline
\end{tabular}

\section{Discussion}

Fungal PJIs are uncommon and extremely challenging regarding their management [5,6]. Most such infections are caused by Candida species [5,35]. Hence, limited data and information exist regarding PJIs caused by non-Candida organisms [8]. The present study aims, by reviewing published data, to clarify the characteristics, treatment options, and outcomes of non-Candida fungal PJIs.

PJIs, along with other invasive fungal infections, represent a major cause of morbidity and mortality in current medical practice. Optimal treatment of fungal PJIs remains unclear, since no certain guidelines exist regarding the antifungal regimen and the indicated surgical intervention [7,8]. TSRA and long-term AFT are proposed due to lack of data $[5,7,8]$. Information about the kind of AFT, its duration, and its success rate, as well as type of surgical intervention, the use of antifungal agents in cement, and the time intervals between the two stages of TSRA, are of utmost importance for the clarification of the best medical treatment and the improvement of the surgical management of these cases.

The present study has reviewed 42 cases of non-Candida fungal PJIs over 37 years, with a mean follow-up of 44.2 months. The incidence of fungal PJIs incidence is expected to rise, due to the increasing number of prosthetic joint reconstruction surgeries worldwide [1,8]. Immunosuppression and systemic disease have been widely acknowledged as risk factors for invasive fungal infections, while revision joint surgeries increase the risk of infection [5]. In the present study, a total of 10 patients $(23.8 \%)$ were immunocompromised, while in $38 \%$ of the cases a revision reconstruction surgery had been performed.

Fungal PJI is frequently of hematogenous origin $[8,14,16]$. However, intraoperative contamination by fungal skin pathogens may also occur [36]. If the contamination originates in the skin, symptoms usually appear early in the postoperative period. In the studied cases, the mean time interval between initial joint reconstruction surgery and onset of symptoms was 42.1 months (ranging from 0.7 to 120), supporting the theory of hematogenous spread. The mean time from onset of symptoms to firm diagnosis was 
5.8 months. Fungal PJIs usually present with indolent symptoms and, therefore, diagnosis may be delayed.

In the present review, the predominant fungi involved were Aspergillus spp., identified in 10 cases $(23.8 \%)$, followed by Coccidioides spp. in 7 cases $(16.7 \%)$ and Pichia anomala in 5 $(11.9 \%)$. In 12 cases (28.6\%), co-infection with bacteria was diagnosed. Concomitant bacterial infection has already been reported in 15-20\% of fungal PJI cases [37]. Coccidioides spp. are geographically restricted dimorphic fungi represented rather heavily in the present review. However, it must be noted that most of the published cases originate in the United States, where these fungi are endemic.

Invasive Aspergillus infections are typically seen in patients with significant underlying immunosuppression [13,14,17]. However, in the present review, most of the reported Aspergillus PJIs occurred in immunocompetent individuals.

Several surgical intervention options have been described for the treatment of fungal PJIs $[7,8]$. In the reviewed population, TSRA was preferred in most cases $(52.4 \%)$, with a success rate of $66.7 \%$. The mean time between the two surgical stages was 5.2 months, and in nine cases cement impregnated with an antifungal agent was used. ORSA, arthrodesis, and debridement each exhibited a $66.7 \%$ success rate, while for RA it was $50 \%$. Eradication of the infection was the result in one case of amputation.

It is of note that arthrodesis, RA, and amputation, although proven to be successful treatment options-since the infection was finally eradicated-are associated with a high negative impact on the patient's quality of life, and may possibly lead to loss of independence. Furthermore, in the eight cases that did not receive any surgical intervention (cases $15,17,19,21,22,24,27$, and 40, shown in Tables 1 and 2), although the treatment's outcome was considered by authors to be successful, all patients subsequently commenced lifelong fungal suppression; therefore, it is understood that definitive eradication of the infection was not achieved. These reports raise the question of whether lifelong fungal suppression by AFT is a feasible option in cases where further surgery may lead to failure, either due to technical difficulties (e.g., extensive bone loss, challenging reconstructive options) or to patient comorbidities.

Guidelines for the treatment of fungal osteoarticular infections exist; however, no clear recommendations are available for the treatment of such PJIs [7]. Therefore, the duration of treatment is mainly based on the clinical and laboratory findings of each case and the physicians' experience with such infections. It is, therefore, of utmost importance to carry out susceptibility testing to obtain accurate MIC values following the isolation of the fungus, taking into account that different species of fungi (e.g., yeast, molds, etc.) are characterized by intrinsic resistance to certain antifungal compounds [8]. Additionally, it must be noted that for a number of molds, laboratory methods indicating MICs are not standardized and unanimously accepted, while the immune status of the patient plays a major role [38].

In all of the reviewed cases, the causative fungus was identified. However, the microbiological procedures used were not described in most of them. Moreover, since the present review spans almost four decades, it is understandable that identification techniques have evolved over time, ranging from simple microscopy, pathology, and culture to modern molecular methods [38]. Hence, although the information about the fungal cause of cases is sufficient, information about specific identification procedures has been inadequate.

Regarding AFT, fluconazole was the preferred antifungal agent (47.6\%), followed by amphotericin B (42.9\%). Fluconazole was extensively used in the reviewed cases, although this agent is ineffective against molds. However, it must be taken into account that fluconazole and amphotericin B deoxycholate were the only available agents in the early years of the reviewed cases. Fluconazole has on rare occasions been associated with severe hepatotoxicity [39]. Hence, liver function tests should be performed regularly during prolonged fluconazole therapy, while amphotericin B, although an effective broad spectrum regimen, is relatively toxic, and its side effects-including renal dysfunction-may restrict 
its long-term use, which is essential for PJI cases [40]. The liposomal compounds of amphotericin B have reduced the drug's nephrotoxicity considerably, but long-term use of these agents may be still problematic [40]. Voriconazole, which was introduced in 2003, has proven to be the drug of choice against Aspergillus spp. This agent has changed the management of Aspergillus infections dramatically over the past several decades; with all the characteristics of azole compounds, it is moderately hepatotoxic and much less nephrotoxic than all amphotericin compounds [41].

The mean AFT duration was found to be 9.4 months. The final outcome was successful in 33 cases $(78.6 \%)$. It is of note, however, that the success rate drops to $66.7 \%$ in cases of bacterial co-infection. Concomitant bacterial infections occur in between 15 and $20 \%$ of fungal cases, while the poor prognosis for co-infective PJIs has already been underlined [42].

The present review has some limitations. There is heterogeneity between different joint reconstruction surgeries (hip, knee, and elbow), while not all information was available from each case, such as specific signs and symptoms of the infection, antifungal dosages, mode of administration, monitoring of serum levels, MICs, and adverse events. Another point not clarified is the specific microbiological techniques used for fungal identification (especially modern ones, such as PCR and/or $\beta$-D-glucan). Nevertheless, this study reviews all of the non-Candida fungal PJIs in a systematic way, offering valuable insights regarding epidemiology, severity, surgical management, and medical treatments that changed dramatically over the long study period, as well as outcomes.

It is important that over the past several decades a number of new antifungal agents (e.g., voriconazole, posaconazole, echinocandins, and isavuconazole) were introduced, providing more medical treatment options, hopefully associated with better results [41].

The present review shows that non-Candida fungal PJIs represent a very challenging clinical entity. A combination of the proper medical AFT, based on susceptibility testing (when feasible) and surgical intervention, seems to represent the current standard management. There have been reports of successful treatment of such cases with OSRA and debridement. However, TSRA should be strongly recommended. The combination of TSRA separated by 3-6 months and a prolonged period of AFT is suggested on the basis of limited data. Lifelong fungal suppression with a proper agent (based on the type of the fungal species) is suggested in cases where surgery is not desirable (either due to patient comorbidities or technical difficulties predicting uncertain results). More data and research are needed, focusing on proper treatment-since the results of therapeutic procedures and policies, such as the antifungal-loaded cement spacers and AFT duration, remain unclear-in order to conclude the optimal management approach.

Author Contributions: Conceptualization, C.K., K.A., G.S.; methodology, C.K., I.C. and S.Z.; validation, C.K., G.V., D.P.K., G.S.; formal analysis, C.K., I.C.; investigation, I.C., S.Z., K.A.; writing—original draft preparation, C.K., I.C.; writing-review and editing, K.R., K.A., G.V., D.P.K., G.S.; supervision, K.A., D.P.K., G.S. All authors have read and agreed to the published version of the manuscript.

Funding: This research received no external funding.

Institutional Review Board Statement: Not applicable, the present study is a literature review.

Informed Consent Statement: Not applicable, the present study is a literature review.

Data Availability Statement: Not applicable.

Acknowledgments: The present review was supported by the Hellenic Society of Medical Mycology.

Conflicts of Interest: The authors declare no conflict of interest.

\section{References}

1. Kurtz, S.; Ong, K.; Lau, E.; Mowat, F.; Halpern, M. Projections of Primary and Revision Hip and Knee Arthroplasty in the United States from 2005 to 2030. J. Bone Jt. Surg. Am. 2007, 89, 780-785. [CrossRef]

2. Besiris, G.T.; Koutserimpas, C.; Karamitros, A.; Karaiskos, I.; Tsakalou, D.; Raptis, K.; Kourelis, K.; Paxinos, O.; Kotsirakis, A.; Vlasis, K. Topical use of tranexamic acid in primary total knee arthroplasty: A comparative study. G-Chair 2020, 41, 126-130.

3. Koutserimpas, C.; Dretakis, K. The evolution of robotic arm-assisted arthroplasty in Greece. G Chir. 2020, 41, 73-78. [PubMed] 
4. Dall'Oca, C.; Ricci, M.; Vecchini, E.; Giannini, N.; Lamberti, D.; Tromponi, C.; Magnan, B. Evolution of TKA design. Acta Bio Med. Atenei Parm. 2017, 88, 17-31. [CrossRef]

5. Beam, E.; Osmon, D. Prosthetic Joint Infection Update. Infect. Dis. Clin. N. Am. 2018, 32, 843-859. [CrossRef]

6. Schoof, B.; Jakobs, O.; Schmidl, S.; Klatte, T.O.; Frommelt, L.; Gehrke, T.; Gebauer, M. Fungal periprosthetic joint infection of the hip: A systematic review. Orthop. Rev. 2015, 7, 5748. [CrossRef] [PubMed]

7. Pappas, P.G.; Kauffman, C.A.; Andes, D.R.; Clancy, C.J.; Marr, K.A.; Ostrosky-Zeichner, L.; Reboli, A.C.; Schuster, M.G.; Vazquez, J.A.; Walsh, T.J.; et al. Executive Summary: Clinical Practice Guideline for the Management of Candid-iasis: 2016 Update by the Infectious Diseases Society of America. Clin. Infect. Dis. 2016, 62, 409-417. [CrossRef]

8. Koutserimpas, C.; Zervakis, S.G.; Maraki, S.; Alpantaki, K.; Ioannidis, A.; Kofteridis, D.P.; Samonis, G. Non-albicans Candida prosthetic joint infections: A systematic review of treatment. World J. Clin. Cases 2019, 7, 1430-1443. [CrossRef] [PubMed]

9. Wu, C.-Y.; Huang, H.-K.; Wu, P.-K.; Chen, W.-M.; Lai, M.-C.; Chung, L.-H. Acremonium Species Combined with Penicillium Species Infection in Hip Hemiarthroplasty: A Case Report and Literature Review. HIP Int. 2014, 24, 656-659. [CrossRef]

10. Gao, Z.; Li, X.; Du, Y.; Peng, Y.; Wu, W.; Zhou, Y. Success Rate of Fungal Peri-Prosthetic Joint Infection Treated by 2-Stage Revision and Potential Risk Factors of Treatment Failure: A Retrospective Study. Med. Sci. Monit. 2018, 24, 5549-5557. [CrossRef]

11. Brown, T.; Petis, S.M.; Osmon, D.R.; Mabry, T.M.; Berry, D.J.; Hanssen, A.D.; Abdel, M.P. Periprosthetic Joint Infection with Fungal Pathogens. J. Arthroplast. 2018, 33, 2605-2612. [CrossRef]

12. Geng, L.; Xu, M.; Yu, L.; Li, J.; Zhou, Y.; Wang, Y.; Chen, J. Risk factors and the clinical and surgical features of fungal prosthetic joint infections: A retrospective analysis of eight cases. Exp. Ther. Med. 2016, 12, 991-999. [CrossRef] [PubMed]

13. Austin, K.S.; Testa, N.; Luntz, R.K.; Greene, J.B.; Smiles, S. Aspergillus infection of total knee arthroplasty presenting as a popliteal cyst: Case report and review of the literature. J. Arthroplast. 1992, 7, 311-314. [CrossRef]

14. Baumann, P.A.; Cunningham, B.; Patel, N.S.; Finn, H.A. Aspergillus fumigatus infection in a mega prosthetic total knee arthroplasty. J. Arthroplast. 2001, 16, 498-503. [CrossRef] [PubMed]

15. Yilmaz, M.; Mete, B.; Ozaras, R.; Kaynak, G.; Tabak, F.; Tenekecioglu, Y.; Öztürk, R.; Y1lmaz, M. Aspergillus fumigatus infection as a delayed manifestation of prosthetic knee arthroplasty and a review of the literature. Scand. J. Infect. Dis. 2011, 43, 573-578. [CrossRef]

16. Hwang, B.-H.; Yoon, J.-Y.; Nam, C.-H.; Jung, K.-A.; Lee, S.-C.; Han, C.-D.; Moon, S.-H. Fungal peri-prosthetic joint infection after primary total knee replacement. J. Bone Jt. Surgery. Br. 2012, 94, 656-659. [CrossRef] [PubMed]

17. Bartash, R.; Guo, Y.; Pope, J.B.; Levi, M.H.; Szymczak, W.; Saraiya, N.; Nori, P. Periprosthetic hip joint infection with Aspergillus terreus: A clinical case and a review of the literature. Med. Mycol. Case Rep. 2017, 18, 24-27. [CrossRef]

18. Kwong, C.A.; Puloski, S.K.T.; Hildebrand, K.A. Fungal periprosthetic joint infection following total elbow arthroplasty: A case report and review of the literature. J. Med. Case Rep. 2017, 11, 20. [CrossRef]

19. Johannsson, B.; Callaghan, J.J. Prosthetic hip infection due to Cryptococcus neoformans: Case report. Diagn. Microbiol. Infect. Dis. 2009, 64, 76-79. [CrossRef]

20. Shah, N.B.; Shoham, S.; Nayak, S. Cryptococcus neoformans Prosthetic Joint Infection: Case Report and Review of the Literature. Mycopathologia 2014, 179, 275-278. [CrossRef] [PubMed]

21. Kuberski, T.; Ianas, V.; Ferguson, T.; Nomura, J.; Johnson, R. Treatment of Prosthetic Joint Infections Associated With Coccidioidomycosis. Infect. Dis. Clin. Pract. 2011, 19, 252-255. [CrossRef]

22. Austen, S.; Van Der Weegen, W.; Verduin, C.M.; Van Der Valk, M.; Hoekstra, H.J. Coccidioidomycosis Infection of a Total Knee Arthroplasty in a Nonendemic Region. J. Arthroplast. 2013, 28, 375.e13-375.e15. [CrossRef]

23. Arbeloa-Gutierrez, L.; Kuberski, T.; Johnson, S.M.; Sagastibelza, I.; Alaez, J.I.; Pappagianis, D. Reactivation of coccidioidomycosis: A prosthetic joint infection in Spain. Eur. J. Clin. Microbiol. Infect. Dis. 2015, 35, 183-186. [CrossRef]

24. Fowler, J.V.G.; Nacinovich, F.M.; Alspaugh, J.A.; Corey, G.R.; Fowler, V.G. Prosthetic joint infection due to Histoplasma capsulatum: Case report and review. Clin. Infect. Dis. 1998, 26, 1017. [CrossRef]

25. Nowbakht, C.; Garrity, K.; Webber, N.; Eraso, J.; Ostrosky-Zeichner, L. Prosthetic Joint Infection Due to Histoplasma capsulatum Complicating a Total Knee Arthroplasty. Open Forum Infect. Dis. 2017, 4, 118. [CrossRef]

26. Leylabadlo, H.E.; Zeinalzadeh, E.; Akbari, N.A.R.; Kafil, H.S. Malassezia species infection of the synovium after total knee arthroplasty surgery. GMS Hyg. Infect. Control 2016, 11, 19.

27. Anagnostakos, K.; Kelm, J.; Schmitt, E.; Jung, J. Fungal Periprosthetic Hip and Knee Joint Infections. J. Arthroplast. 2012, 27, 293-298. [CrossRef] [PubMed]

28. Wang, Q.-J.; Shen, H.; Zhang, X.-L.; Jiang, Y.; Wang, Q.; Chen, Y.; Shao, J.-J. Staged reimplantation for the treatment of fungal peri-prosthetic joint infection following primary total knee arthroplasty. Orthop. Traumatol. Surg. Res. 2015, 101, 151-156. [CrossRef] [PubMed]

29. Gottesman-Yekutieli, T.; Dan, M.; Shwartz, O.; Edelman, A.; Hendel, D. Pseudallescheria boydii Infection of a Prosthetic Hip Joint-An Uncommon Infection in a Rare Location. Am. J. Med. Sci. 2011, 342, 250-253. [CrossRef] [PubMed]

30. Lackner, M.; De Man, F.H.; Eygendaal, D.; Wintermans, R.G.F.; Kluytmans, J.A.; Klaassen, C.H.; Meis, J.F. Severe prosthetic joint infection in an immunocompetent male patient due to a therapy refractory Pseudallescheria apiosperma. Mycoses 2011, 54, 22-27. [CrossRef]

31. Savini, V.; Sozio, F.; Catavitello, C.; Talia, M.; Manna, A.; Febbo, F.; Balbinot, A.; Di Bonaventura, G.; Piccolomini, R.; Parruti, G.; et al. Femoral Prosthesis Infection by Rhodotorula mucilaginosa. J. Clin. Microbiol. 2008, 46, 3544-3545. [CrossRef] 
32. Dehart, D.J. Use of Itraconazole for Treatment of Sporotrichosis Involving a Knee Prosthesis. Clin. Infect. Dis. 1995, 21, 450. [CrossRef] [PubMed]

33. Ceffa, R.; Andreoni, S.; Borrè, S.; Ghisellini, F.; Fornara, P.; Brugo, G.; Ritter, M.A. Mucoraceae infections of antibiotic-loaded cement spacers in the treatment of bacterial infections caused by knee arthroplasty. J. Arthroplast. 2002, 17, 235-238. [CrossRef] [PubMed]

34. Zuo, Q.; Dong, L.; Mu, W.; Zhou, L.; Hu, T.; Zhang, H. Trichosporon asahii Infection after Total Knee Arthroplasty: A Case Report and Review of the Literature. Can. J. Infect. Dis. Med. Microbiol. 2015, 26, 47-51. [CrossRef]

35. Koutserimpas, C.; Samonis, G.; Velivassakis, E.; Iliopoulou-Kosmadaki, S.; Kontakis, G.; Kofteridis, D.P. Candida glabrata prosthetic joint infection, successfully treated with anidulafungin: A case report and review of the literature. Mycoses 2017, 61, 266-269. [CrossRef] [PubMed]

36. Nucci, M.; Anaissie, E. Revisiting the Source of Candidemia: Skin or Gut? Clin. Infect. Dis. 2001, 33, 1959-1967. [CrossRef]

37. Tande, A.J.; Patel, R. Prosthetic Joint Infection. Clin. Microbiol. Rev. 2014, 27, 302-345. [CrossRef]

38. Lamoth, F.; Lewis, R.E.; Kontoyiannis, D.P. Role and Interpretation of Antifungal Susceptibility Testing for the Management of Invasive Fungal Infections. J. Fungi 2020, 7, 17. [CrossRef] [PubMed]

39. Pasternak, B.; Wintzell, V.; Furu, K.; Engeland, A.; Neovius, M.; Stephansson, O. Oral Fluconazole in Pregnancy and Risk of Stillbirth and Neonatal Death. JAMA 2018, 319, 2333-2335. [CrossRef]

40. Hamill, R.J. Amphotericin B Formulations: A Comparative Review of Efficacy and Toxicity. Drugs 2013, 73, 919-934. [CrossRef] [PubMed]

41. Nett, J.E.; Andes, D.R. Antifungal Agents. Infect. Dis. Clin. N. Am. 2016, 30, 51-83. [CrossRef] [PubMed]

42. Sidhu, M.S.; Cooper, G.; Jenkins, N.; Jeys, L.; Parry, M.; Stevenson, J.D. Prosthetic fungal infections. Bone Jt. J. 2019, 101-B, 582-588. [CrossRef] [PubMed] 